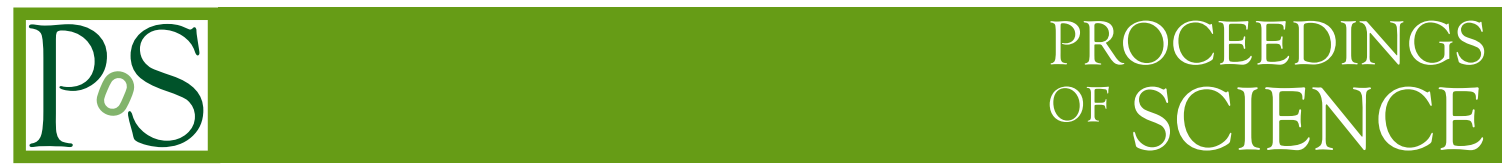

\title{
Diboson and multiboson results with ATLAS
}

\section{Sarah Louise Barnes* ${ }^{\dagger}$}

University of Manchester (UK)

E-mail: sarah.barnesecern.ch

Measurements of electroweak gauge-boson pair-production and multi-boson production at $\sqrt{s}=$ $8 \mathrm{TeV}$ and $\sqrt{s}=13 \mathrm{TeV}$ in $p p$ collisions with the ATLAS detector are presented. Measurements of $W W, W Z, Z \gamma$ and $Z \gamma \gamma$ production at $\sqrt{s}=8 \mathrm{TeV}$ are presented along with measurements of $Z Z$ and $W Z$ production at $\sqrt{s}=13 \mathrm{TeV}$. Limits on anomalous gauge boson couplings are given, along with total, fiducial and differential cross-section measurements.

Fourth Annual Large Hadron Collider Physics

13-18 June 2016

Lund, Sweden

* Speaker.

$\dagger$ on behalf of the ATLAS Collaboration 


\section{Introduction}

The study of vector-boson pair production with the ATLAS experiment [1] at the LHC provides an important test of the Standard Model (SM) at the TeV scale. In addition, an understanding of such processes allows for a precise background estimation needed for many measurements. Finally, the study of multi-boson production allows constraints to be set on many exotic models through the study of cross-sections and triple and quartic gauge boson couplings (TGC's and QGC's).

The multi-boson measurement strategy at the ATLAS experiment consists of four main measurements. The first being the measurement of the fiducial cross-section. This is defined in equation 1.1, where $N_{\text {data }}$ is the number of observed events, $N_{b g}$ is the number of expected background events, $\mathscr{L}$ is the integrated luminosity and $C_{V V}$ is the ratio of the number of measured to the generated number of events in the fiducial region.

$$
\sigma_{f i d}=\frac{N_{d a t a}-N_{b g}}{\mathscr{L} C_{V V}}
$$

The fiducial cross-section can be extrapolated to the total phase space through the inclusion of one extra factor, $A_{V V} \mathscr{B}$. This is shown in equation 1.2, where $A_{V V}$ is the ratio of events in the fiducial region to all generated events and $\mathscr{B}$ is the branching ratio of the process.

$$
\sigma_{t o t}=\sigma_{f i d} \times \frac{1}{A_{V V} \mathscr{B}}
$$

Unfolded differential distributions are also studied. Finally, high momentum events are probed to set limits on anomalous TGC's and QGC's (aTGC's and aQGC's). In the SM only charged couplings are allowed, neutral couplings are not allowed. Any observation of additional couplings would indicate the presence of new physics.

One method used to study anomalous gauge boson couplings (aGCs) is through an effective Lagrangian approach [2]. This probes for new physics at an energy scale $\Lambda$ in a model independent way. The effective Lagrangian is defined in equation 1.3, where $\alpha_{i}^{(n)}$ are coupling coefficients and $\mathscr{O}_{i}^{(n)}$ are operators of dimension mass $^{4+n}$.

$$
\mathscr{L}=\sum_{n} \frac{1}{\Lambda^{n}} \sum_{i} \alpha_{i}^{(n)} \mathscr{O}_{i}^{(n)}
$$

When expanding up to dimension 6 and including only CP conserving couplings the following expression is obtained

$$
\mathscr{L}=-i g_{W W V}\left[g_{1}^{V}\left(W_{\mu \nu}^{\dagger} W^{\mu}-W^{\dagger \mu} W_{\mu v}\right) V^{v}+\kappa^{V} W_{\mu}^{\dagger} W_{v} V^{\mu v}+\frac{\lambda_{V}}{m_{W}^{2}} W_{\lambda \mu}^{\dagger} W_{v}^{\mu} V^{v \lambda}\right],
$$

where the coefficients $g_{1}^{V}, \kappa_{V}$ and $\lambda_{V}$ are defined to be 1,1 and 0 respectively in the SM. Limits on anomalous couplings are set in several different scenarios. In the 'LEP' scenario, the coefficients are defined such that $\Delta \kappa_{\gamma}=\left(\cos ^{2} \theta_{W}\right) /\left(\sin ^{2} \theta_{W}\right)\left(\Delta g_{1}^{Z}-\Delta \kappa_{Z}\right)$ and $\lambda_{Z}=\lambda_{\gamma}$. In the 'HISZ' scenario $\Delta g_{1}^{Z}=\Delta \kappa_{Z} /\left(\cos ^{2} \theta_{W}-\sin ^{2} \theta_{W}\right), \Delta \kappa_{\gamma}=2 \Delta \kappa_{Z} \cos ^{2} \theta_{W} /\left(\cos ^{2} \theta_{W}-\sin ^{2} \theta_{W}\right)$ and $\lambda_{Z}=\lambda_{\gamma}$. Finally, in the 'equal couplings' scenario $\Delta \kappa_{\gamma}=\Delta \kappa_{Z}, g_{1}^{Z}=1$ and $\lambda_{Z}=\lambda_{\gamma}$. An additional factor is often required for each parameter in order to restore unitarity, this factor is of the form $\alpha(\hat{s})=\lambda /\left(1+\hat{s} / \Lambda^{2}\right)^{2}$. 
The following sections will discuss recent di-boson and multi-boson measurements with the ATLAS experiment. Measurements of $W^{+} W^{-} \rightarrow \ell^{+} v \ell^{-} v, W^{ \pm} Z \rightarrow \ell^{ \pm} v \ell^{+} \ell^{-}$and $Z \gamma / Z \gamma \gamma$ (where $Z \rightarrow \ell^{+} \ell^{-}$or $v v$ ) production have been made at $8 \mathrm{TeV}$. Also, measurements of $Z Z \rightarrow \ell^{+} \ell^{-} \ell^{\prime} \ell^{\prime}-$ and $W^{ \pm} Z \rightarrow \ell^{ \pm} v \ell^{+} \ell^{-}$production have been made at $13 \mathrm{TeV}$.

\section{2. $W^{+} W^{-} \rightarrow \ell^{+} v \ell^{-} v$ production at $\sqrt{s}=8 \mathrm{TeV}$}

The $W^{+} W^{-} \rightarrow \ell^{+} v \ell^{-} v$ production cross-section has been measured with $20.3 \mathrm{fb}^{-1}$ of data collected at $\sqrt{s}=8 \mathrm{TeV}$ with the ATLAS detector. In addition unfolded differential distributions have been produced, and limits have been set on aTGC parameters in multiple scenarios [3].

Events are selected with two high transverse momentum $\left(p_{T}\right)$, opposite-charge leptons (electrons or muons). It is required that the invariant mass of the two leptons $\left(m_{l l}\right)$ must satisfy $\mid m_{l l}-$ $m_{Z} \mid>15 \mathrm{GeV}$. Also, events must have exactly zero jets in order to suppress the top quark background. Requirements are also made on $E_{T, R e l}^{\text {miss }}$ which is defined such that

$$
E_{T, R e l}^{\text {miss }}=\left\{\begin{array}{ll}
E_{T}^{\text {miss }} \times \sin \Delta \phi_{\ell} & \text { if } \quad \Delta \phi_{\ell}<\pi / 2 \\
E_{T}^{\text {miss }} & \text { if } \Delta \phi_{\ell}<\pi / 2
\end{array},\right.
$$

where $E_{T}^{\text {miss }}$ is the missing transverse momentum in the event and $\Delta \phi_{l}$ is the difference in azimuthal angle $\phi$ between $\mathbf{E}_{\mathbf{T}}^{\text {miss }}$ and the nearest lepton. The bold notation indicates a vector. It is required that $E_{T, R e l}^{\text {miss }}>15 \mathrm{GeV}$ for the $e \mu$ final state and $E_{T, R e l}^{\text {miss }} \geq 45 \mathrm{GeV}$ for the $e e / \mu \mu$ final states. This is applied in order to reduce the contribution from the Drell-Yan background.

A total of 6636 events were selected with a total expectation of $5747 \pm 473$ signal plus background events. The dominant background contribution is from the top-quark background, which is estimated using a data driven approach. The background contribution is of the order $20-30 \%$.

The fiducial cross-section measurements are shown for each final state in Figure 1 (a), compared to the corresponding theoretical prediction. Figure 1 (b) shows the total cross-section measurements compared with the theretocal predictions. The total cross-section was measured to be $\sigma_{t o t}\left(W^{+} W^{-} \rightarrow \ell^{+} v \ell^{-} v\right)=71.1 \pm 1.1$ (stat.) ${ }_{-5.0}^{+5.7}$ (syst.) \pm 1.4 (lumi.) pb, which can be compared to the next-to-next-to-leading order (NNLO) theoretical prediction of $\sigma_{\text {Theory }}\left(W^{+} W^{-} \rightarrow \ell^{+} v \ell^{-} v\right)=$ $63.2 \pm_{-1.4}^{+1.6}$ (scale) \pm 1.2 (PDF) pb. The systematic uncertainties dominate the overall uncertainty on the cross-section, with the largest contributions coming from the uncertainty on the jet energy scale (4\%) and the knowledge of the $W+$ jets background (3\%). It is evident that in all cases the measurements lie slightly higher than the theoretical prediction by around $1-2 \sigma$.

The unfolded differential cross-section for the $p_{T}$ of the highest momentum (leading) lepton is shown in Figure 2. Good agreement can be seen between the shape of the data and the prediction, although the prediction slightly undershoots the data.

Finally, limits on aTGC parameters were set. The limits in the 'LEP' scenario are shown in Figure 3 compared to the limits on the charged couplings from ATLAS [5] [4], CMS [6], D0 [7] and the LEP combination [8]. It is evident that the limits set from this analysis are consistent with the SM and are the most stringent limits available at this time. Limits are shown for $\Lambda=\infty$ and are set by fitting the leading lepton $p_{T}$ distribution. 


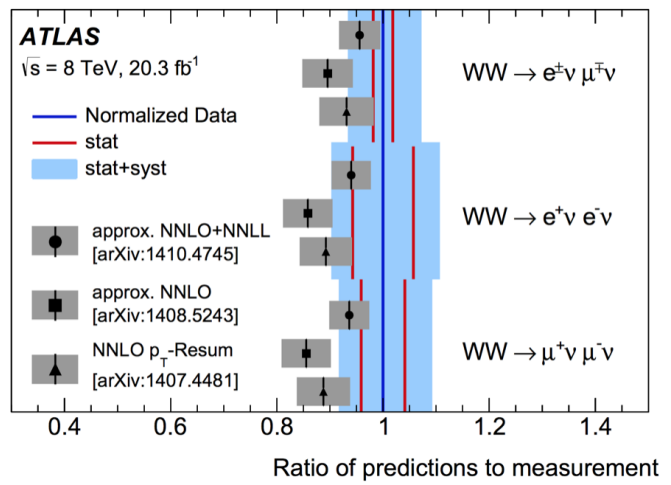

(a)

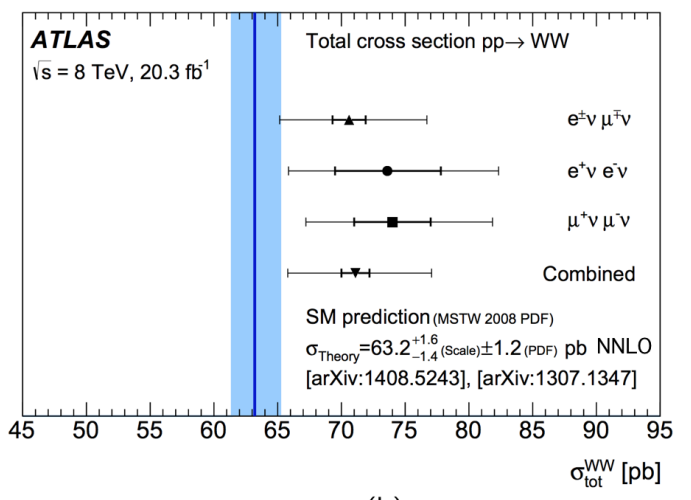

(b)

Figure 1: The fiducial (a) and total (b) cross-section measurements compared with the NNLO theoretical predictions. The theoretical predictions are shown by the grey points for (a) and by the blue band in (b). The measurement is shown by the blue line in (a), with the blue band representing the total uncertainty. In (b) the measurements are shown by the black points with the total uncertainties represented by the error bar [3].

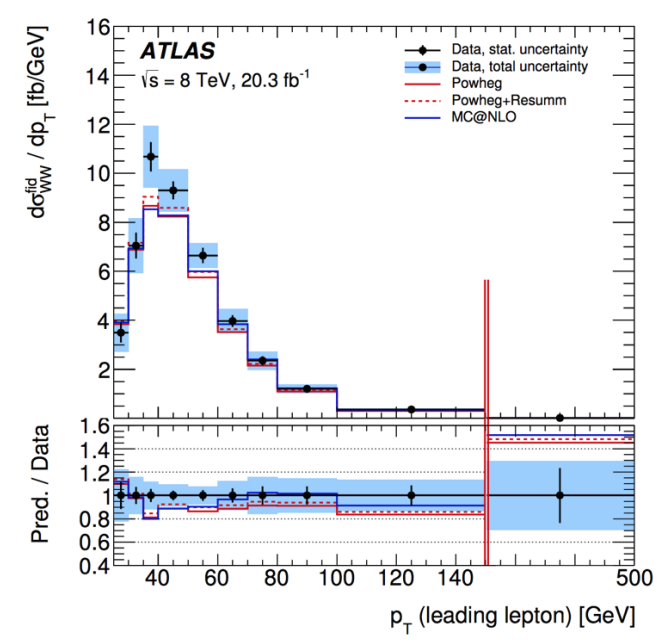

Figure 2: The $W W$ differential cross section as a function of the leading lepton $p_{T}$. The theoretical predictions from Powheg are indicated by the red and red-dashed lines and the predictions from MC@NLO are shown by the blue lines. The data points are shown in black and the total uncertainty is shown by the blue band. The lower panel shows the ratio of the MC predictions to the data. [3].

\section{3. $W^{ \pm} Z \rightarrow \ell^{ \pm} v \ell^{+} \ell^{-}$production at $\sqrt{s}=8 \mathrm{TeV}$}

For the $W^{ \pm} Z \rightarrow \ell^{ \pm} v \ell^{+} \ell^{-}$analysis an equivalent set of measurements to the $W W$ analysis have been made. These measurements also use $20.3 \mathrm{fb}^{-1}$ of data collected at $\sqrt{s}=8 \mathrm{TeV}$ [4].

Events are required to have exactly three high $p_{T}$ leptons, this is applied in order to reduce the $Z Z$ background contribution. Two of the leptons are required to have an invariant mass such that $\left|m_{l l}-m_{Z}\right|<10 \mathrm{GeV}$. The final lepton is assigned to the $W$ boson, which is required to have a transverse mass $\left(m_{T}^{W}\right)>30 \mathrm{GeV}$. The transverse mass is defined to be $m_{T}=\sqrt{2\left(E_{T}^{\ell} E_{T}^{v}-\vec{p}_{T}^{\ell} \vec{p}_{T}^{v}\right)}$. A total of 2091 events were selected with a total prediction of $1824.8 \pm 7.0$ signal plus background 


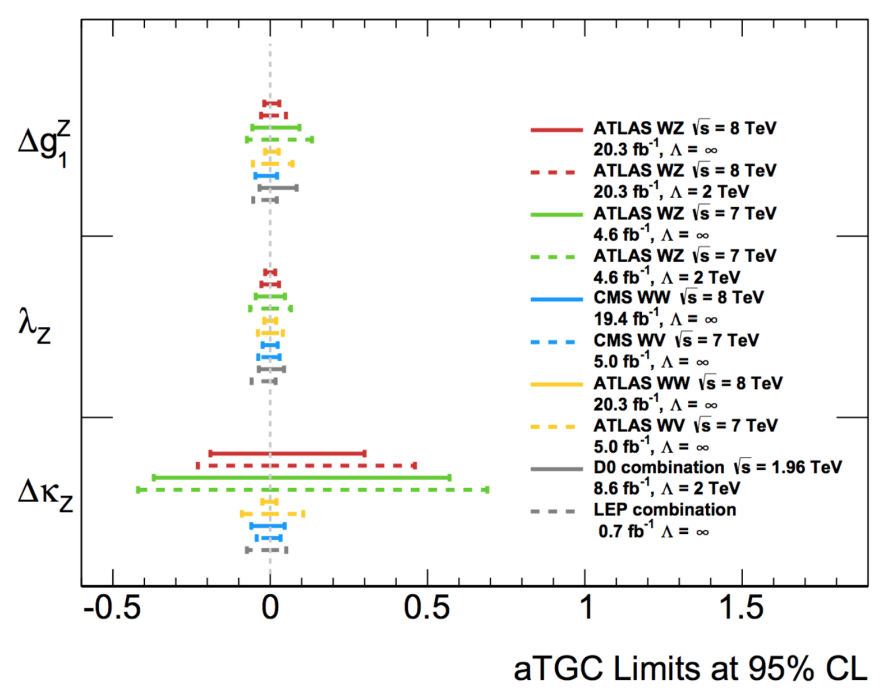

Figure 3: Comparison of aTGC limits set in $W W$ and $W Z$ events at the ATLAS [5] [4], CMS [6] and D0 experiments [7] along with the LEP combination [8]. The results from the $W W$ and $W Z$ analyses at $8 \mathrm{TeV}$ are shown by the yellow solid line and red lines respectively [4].

events. The dominant backgrounds for this process come from $Z Z$ production and from non-prompt sources. The $Z Z$ background is estimated from Monte-Carlo (MC) and the non-prompt contribution is estimated using a data-driven approach. The $W^{ \pm} Z$ fiducial cross-section measurements compared with the Powheg NLO theoretical prediction are shown in Figure 4 (a), along with the $W^{+} Z / W^{-} Z$ ratio in Figure 4 (b). The total cross-section for all final states combined was calculated to be $\sigma_{t o t}\left(W^{ \pm} Z \rightarrow \ell^{ \pm} v \ell^{+} \ell^{-}\right)=24.3 \pm 0.6$ (stat.) \pm 0.6 (syst.) \pm 0.4 (lumi.) pb., this can be compared to the theoretical prediction of $20.98 \pm 0.02$ (stat.) \pm 0.70 (PDF.) \pm 0.90 (scale.) pb. The measured fiducial and total cross-sections are both higher than the theoretical predictions by around $10 \%$. The theoretical prediction, however, is calculated at NLO. Recently a NNLO prediction has become available [9], when comparing the measurements to the NNLO prediction the agreement is significantly improved. This will be discussed further in section 6 . The uncertainties on the cross-section measurements have equal contributions from the statistics and the systematics. The dominant systematic uncertainties arise due to the electron and muon identification efficiencies (1\% and $1.4 \%$ respectively) as well as the knowledge of the non-prompt background (1.3\%).

Differential cross-section measurements were also presented. Figure 5 shows the differential cross-section for the $m_{T}^{W Z}$ variable, which was also used to set limits on aTGC's. From Figure 5 it is evident that in general there is good agreement between the shape of the data and the MC. The aTGC limits for the 'LEP' scenario are shown in Figure 3, and can be seen to be consistent with the SM predictions.

\section{4. $Z \gamma$ and $Z \gamma \gamma$ production at $\sqrt{s}=8 \mathrm{TeV}$}

For the $Z \gamma$ and $Z \gamma \gamma$ analyses, leptonic decay modes of the $Z$ boson are considered ( $Z \rightarrow$ $e^{+} e^{-}, \mu^{+} \mu^{-}$or $\left.v \bar{v}\right)$. The $Z$ bosons are associated with one or two photons + ' $\mathrm{X}$ ', where ' $\mathrm{X}$ ' is the recoil system. Both an inclusive and exclusive selection is made, the inclusive selection implies 


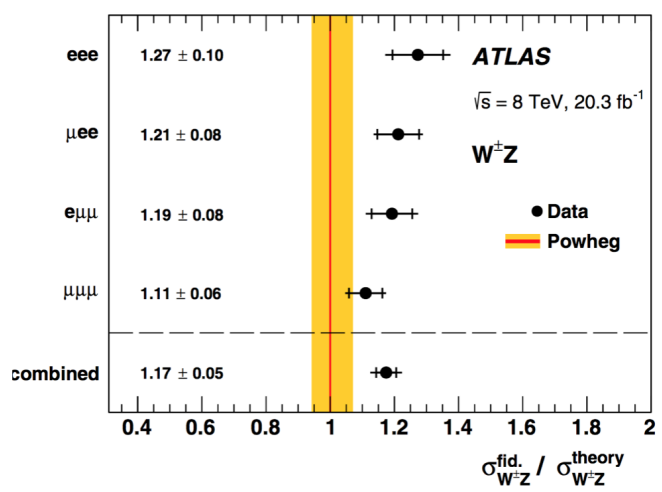

(a)

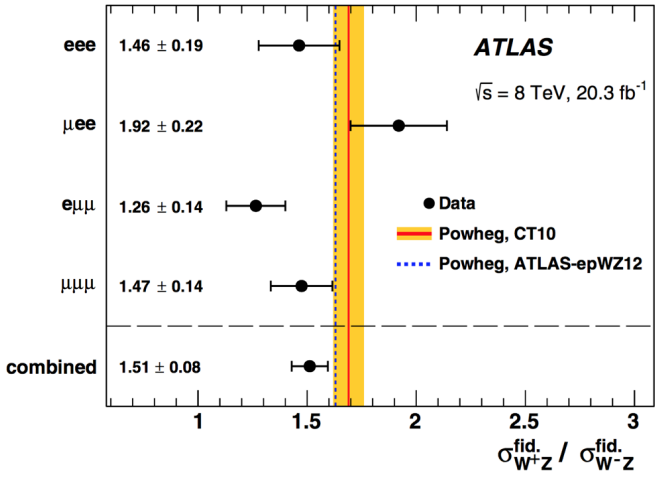

(b)

Figure 4: The $W^{ \pm} Z$ (a) and $W^{+} Z / W^{-} Z$ (b) fiducial cross-section measurements at $8 \mathrm{TeV}$ compared with the Powheg NLO theoretical predictions. The theoretical predictions are indicated by the yellow band and the measurements are indicated by the black points and error bars. In (b) an alternative prediction using the ATLAS-epWZ12 PDF set is shown by the blue dashed line [4].

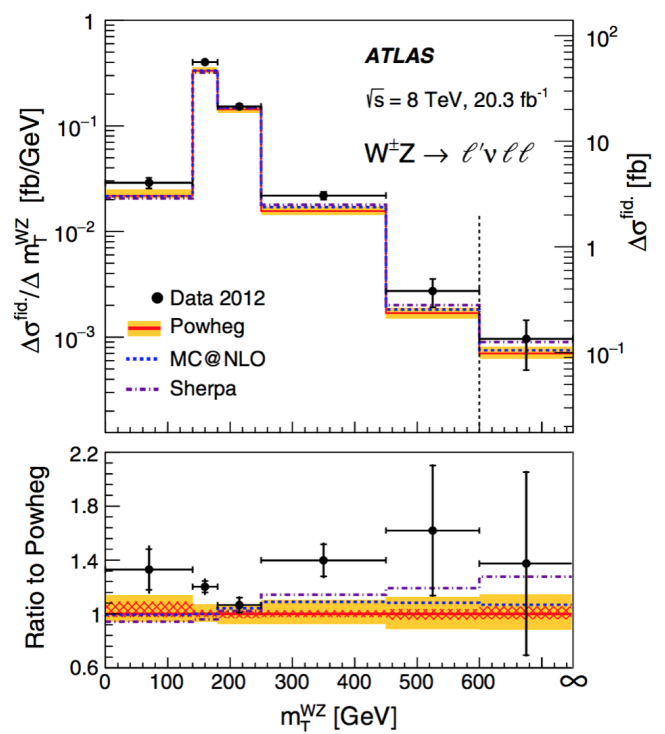

Figure 5: The $W^{ \pm} Z$ differential cross section as a function of $m_{T}^{W Z}$. The theoretical predictions from Powheg (red line), MC@NLO (blue line) and Sherpa (purple line) are shown. The data points are indicated in black and the total uncertainty is indicated by the yellow band. The lower panel shows the ratio of the data, MC@NLO and Shepra to the Powheg prediction [4].

that the number of additional jets observed is $\geq 0$ and for the exclusive case no additional jets are observed. Measurements are made using $20.3 \mathrm{fb}^{-1}$ of data collected at $\sqrt{s}=8 \mathrm{TeV}$ [10]. For the $Z \rightarrow \ell^{+} \ell^{-}$channels events are selected which have two same-flavour opposite-sign leptons with $m_{l l}>40 \mathrm{GeV}$. Events are also required to have high photon transverse energy $E_{T, \gamma}$, with the exact selection depending on the final state. For the $Z \rightarrow v \bar{v}$ channels high $E_{T}^{\text {miss }}$ is required. $\left(E_{T}^{\text {miss }}>100\right.$ $\mathrm{GeV}$ for $Z \gamma$ and $E_{T}^{\text {miss }}>110 \mathrm{GeV}$ for $Z \gamma \gamma$ ). The $E_{T, \gamma}$ selection is summarised in Table 1.

A total of $53867 Z \gamma$ data events were observed, along with $131 Z \gamma \gamma$ events. The dominant 


\begin{tabular}{ccccc}
\hline \hline Selection & $\ell^{+} \ell^{-} \gamma+X$ & $\ell^{+} \ell^{-} \gamma \gamma+X$ & $\nu \bar{v} \gamma+X$ & $\nu \bar{v} \gamma \gamma+X$ \\
\hline$E_{T, \gamma} \mathrm{GeV}$ & 15 & 15 & 130 & 22 \\
\hline
\end{tabular}

Table 1: Requirements on $E_{T, \gamma}$ for the $Z \gamma$ and $Z \gamma \gamma$ analysis.

background for this analysis comes from photon misidentification. Both channels have a significant contribution from the background, this can be seen in Figure 6 which shows the $E_{T}^{\gamma}$ distribution for the $Z\left(e^{+} e^{-}\right) \gamma(\mathrm{a})$ and $Z(v \bar{v}) \gamma(\mathrm{b})$ channels. Good agreement can be seen between data and the MC prediction.

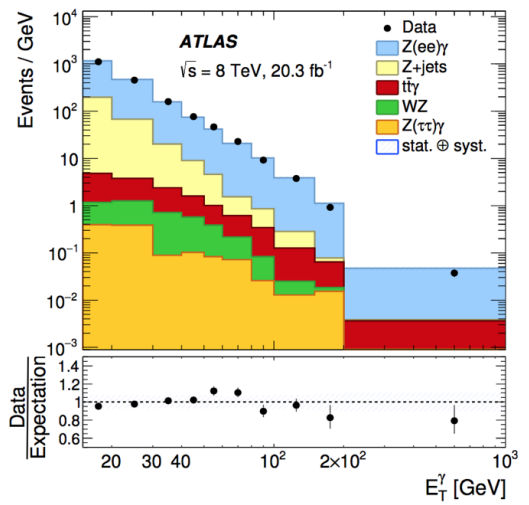

(a)

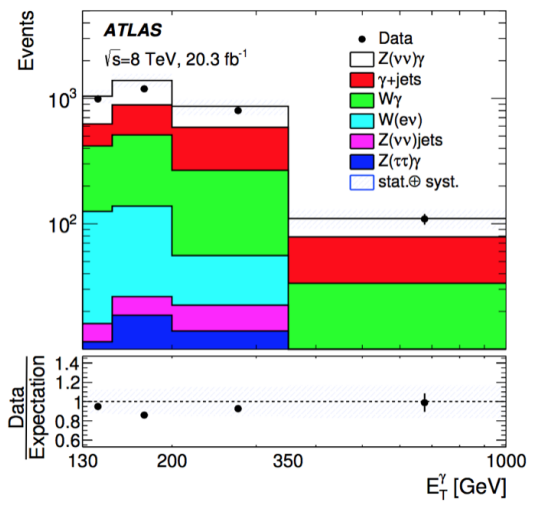

(b)

Figure 6: $E_{T}^{\gamma}$ distributions for the $Z\left(e^{+} e^{-}\right) \gamma($ a) and $Z(v \bar{v}) \gamma($ b) channels. Data is indicated by the black points. The signal prediction is shown in blue (a) and white (b). The Background predictions are indicated by the various coloured histograms. The lower panel shows the ratio of the data to the expectation [10].

For all final states fiducial cross-section measurements were made. Many final states result from this analysis, the cross-section measurements for each are summarised in Figure 7. In all cases there is good agreement between the measurement and the prediction, with a maximum deviation of around $1.7 \sigma$ for the $\ell^{+} \ell^{-} \gamma \gamma$ final state. The uncertainties are generally systematically dominated for the $Z \gamma$ channel and statistically dominated for the $Z \gamma \gamma$ channel. The systematic uncertainties vary depending on the final state but are generally dominated by uncertainties relating to photon identification, electromagnetic energy scale and lepton isolation and impact parameter selection efficiency.

Limits were also set on the neutral aTGC coupling parameters $h_{3}^{\gamma}, h_{4}^{\gamma}, h_{3}^{Z}$ and $h_{4}^{Z}$. Also, limits were set on the aQGC parameters $a_{0}$ and $a_{c}$. These are shown in Figure 8. It can be seen that the aTGC limits are equal to or improving upon the most stringent limits available. Also, the aQGC limits are the first limits to be set on these parameters from $Z \gamma \gamma$ events.

\section{5. $Z Z \rightarrow \ell^{+} \ell^{-} \ell^{\prime}+\ell^{\prime}-$ production at $\sqrt{s}=13 \mathrm{TeV}$}

For the $Z Z \rightarrow \ell^{+} \ell^{-} \ell^{\prime}+\ell^{\prime}-$ analysis measurements are made using $3.2 \mathrm{fb}^{-1}$ of data collected at $\sqrt{s}=13 \mathrm{TeV}$ [11]. Measurements of both the total and fiducial cross-sections were made. Events were selected with exactly two same-flavour opposite-sign pairs of leptons with $66 \mathrm{GeV}<m_{l l}<$ 


\begin{tabular}{|c|c|c|c|}
\hline Channel & Measurement $[\mathrm{fb}]$ & MCFM Prediction [fb] & NNLO Prediction [fb] \\
\hline \multicolumn{4}{|c|}{$N_{\text {jets }} \geq 0$} \\
\hline$e^{+} e^{-} \gamma$ & $1510 \pm 15$ (stat. $)_{-84}^{+91}$ (syst. $)_{-28}^{+30}$ (lumi.) & \multirow{3}{*}{$1345_{-82}^{+66}$} & \multirow{3}{*}{$1483_{-37}^{+19}$} \\
\hline$\mu^{+} \mu^{-} \gamma$ & $1507 \pm 13$ (stat. $)_{-73}^{+78}$ (syst. $)_{-28}^{+29}$ (lumi. $)$ & & \\
\hline$\ell^{+} \ell^{-} \gamma$ & $1507 \pm 10$ (stat. $)_{-73}^{+78}$ (syst. $)_{-28}^{+29}$ (lumi.) & & \\
\hline$v \bar{\gamma} \gamma$ & $68 \pm 4$ (stat.) ${ }_{-32}^{+33}$ (syst.) \pm 1 (lumi.) & $68.2 \pm 2.2$ & $81.4_{-2.2}^{+2.4}$ \\
\hline \multicolumn{4}{|c|}{$N_{\text {jets }}=0$} \\
\hline$e^{+} e^{-} \gamma$ & $1205 \pm 14$ (stat.) ${ }_{-75}^{+84}$ (syst.) \pm 23 (lumi.) & \multirow{3}{*}{$1191_{-89}^{+71}$} & \multirow{3}{*}{$1230_{-18}^{+10}$} \\
\hline$\mu^{+} \mu^{-} \gamma$ & $1188 \pm 12$ (stat.) ${ }_{-63}^{+68}$ (syst.) ${ }_{-22}^{+23}$ (lumi.) & & \\
\hline$\ell^{+} \ell^{-} \gamma$ & $1189 \pm 9$ (stat. $)_{-63}^{+69}$ (syst. $)_{-22}^{+23}$ (lumi.) & & \\
\hline$v \bar{v} \gamma$ & $43 \pm 2$ (stat.) \pm 10 (syst.) \pm 1 (lumi.) & $51.0_{-2.3}^{+2.1}$ & $49.21_{-0.52}^{+0.61}$ \\
\hline \multicolumn{4}{|c|}{$N_{\text {jets }} \geq 0$} \\
\hline$e^{+} e^{-} \gamma \gamma$ & $6.2_{-1.1}^{+1.2}$ (stat.) \pm 0.4 (syst.) \pm 0.1 (lumi.) & \multirow{3}{*}{$3.70_{-0.11}^{+0.21}$} & \\
\hline$\mu^{+} \mu^{-} \gamma \gamma$ & $3.83_{-0.85}^{+0.95}$ (stat.) $)_{-0.47}^{+0.48}$ (syst.) \pm 0.07 (lumi.) & & \\
\hline$\ell^{+} \ell^{-} \gamma \gamma$ & $5.07_{-0.68}^{+0.73}$ (stat. $)_{-0.38}^{+0.41}$ (syst.) \pm 0.10 (lumi. $)$ & & \\
\hline$v \bar{\gamma} \gamma \gamma$ & $2.5_{-0.9}^{+1.0}$ (stat.) \pm 1.1 (syst.) \pm 0.1 (lumi.) & $0.737_{-0.032}^{+0.039}$ & \\
\hline \multicolumn{4}{|c|}{$N_{\text {jets }}=0$} \\
\hline$e^{+} e^{-} \gamma \gamma$ & $4.6_{-0.9}^{+1.0}$ (stat. $)_{-0.3}^{+0.4}$ (syst.) \pm 0.1 (lumi. $)$ & \multirow{3}{*}{$2.91_{-0.12}^{+0.23}$} & \\
\hline$\mu^{+} \mu^{-} \gamma \gamma$ & $2.38_{-0.67}^{+0.77}$ (stat. $)_{-0.32}^{+0.33}$ (syst. ${ }_{-0.04}^{+0.05}$ (lumi.) & & \\
\hline$\ell^{+} \ell^{-} \gamma \gamma$ & $3.48_{-0.56}^{+0.61}$ (stat.) $)_{-0.25}^{+0.29}$ (syst.) \pm 0.07 (lumi. $)$ & & \\
\hline$v \bar{v} \gamma \gamma$ & $1.18_{-0.44}^{+0.52}$ (stat.) $)_{-0.49}^{+0.48}$ (syst.) \pm 0.02 (lumi.) & $0.395_{-0.037}^{+0.049}$ & \\
\hline
\end{tabular}

Figure 7: Measured cross-sections for the $Z \gamma$ and $Z \gamma \gamma$ processes at $\sqrt{s}=8 \mathrm{TeV}$ in multiple fiducial regions defined in reference [10]. The SM predictions at NLO from the MCFM generator are given, along with the NNLO predictions for the $Z \gamma$ channel only [10].

$116 \mathrm{GeV}$. A total of 63 data events were observed, with a small total background contribution of around $1 \%$, dominated by $t t Z$ production and misidentified leptons. The fiducial cross-section measurements are given in Figure 9 (a) and are compared with the NNLO theoretical prediction. The measurements are in good agreement with the prediction. Figure 9 (b) shows the $Z Z$ total cross-section as a function $\sqrt{s}$. In this plot the theoretical prediction shown is calculated using MCFM at NLO. It is evident that all of the measurements at energies between $2-13 \mathrm{TeV}$ lie slightly above the expectation, however are all consistent within the uncertainties. The agreement is likely to be improved when compared to the NNLO prediction. The $Z Z 13 \mathrm{TeV}$ cross-section measurements are currently statistically limited. The dominant systematic uncertainties are due to the lepton reconstruction and identification efficiency scale factors and the difference between the MC generators used to model the signal processes.

\section{6. $W^{ \pm} Z \rightarrow \ell^{ \pm} v \ell^{+} \ell^{-}$production at $\sqrt{s}=13 \mathrm{TeV}$}

Measurements of $W^{ \pm} Z \rightarrow \ell^{ \pm} v \ell^{+} \ell^{-}$production were also made using $3.2 \mathrm{fb}^{-1}$ of data collected at $\sqrt{s}=13 \mathrm{TeV}$ [12]. The event selection applied is as described in section 3. A total of 569 events were observed, with a corresponding expectation of $506 \pm 38$ signal plus background events. As with the $8 \mathrm{TeV}$ analysis, the dominant background processes are from non-prompt sources and $Z Z$ production. The $W^{ \pm} Z$ fiducial cross section measurements are shown in Figure 10 (a), compared with the NLO theoretical prediction from Powheg. Figure 10 (b) shows the ratio of the $W^{+} Z / W^{-} Z$ fiducial cross-sections again compared with the MC prediction. In both cases the 


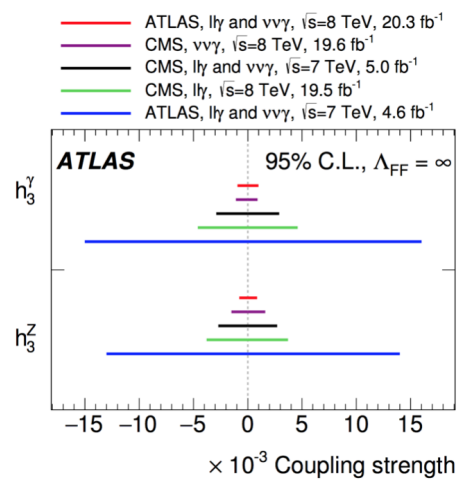

(a)

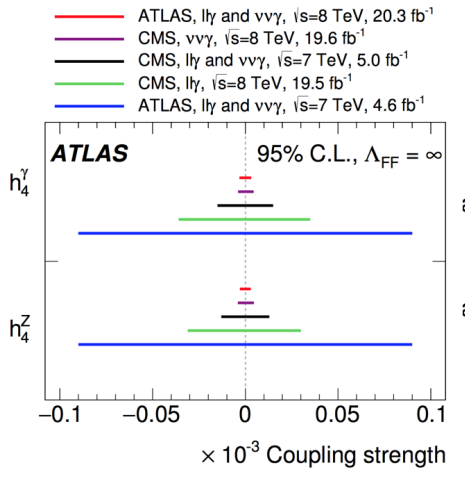

(b)

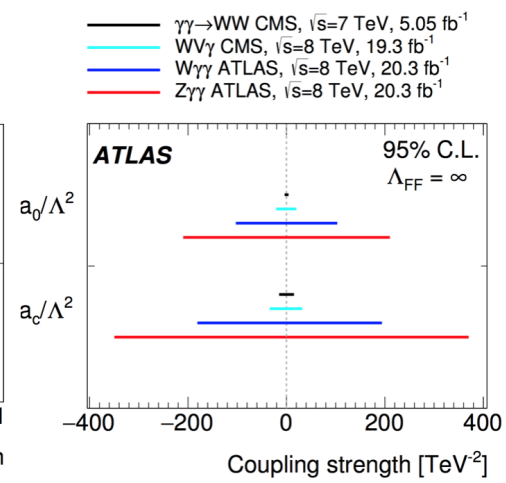

(c)

Figure 8: Limits from the $Z \gamma$ analysis on aTGC parameters $h_{3}^{\gamma}, h_{4}^{\gamma}$ (a) and $h_{3}^{Z}, h_{4}^{Z}$ (b). Also shown are limits from the $Z \gamma \gamma$ analysis on aQGC parameters $a_{0}$ and $a_{c}$ (c). The limits from these analyses are shown in red [10].

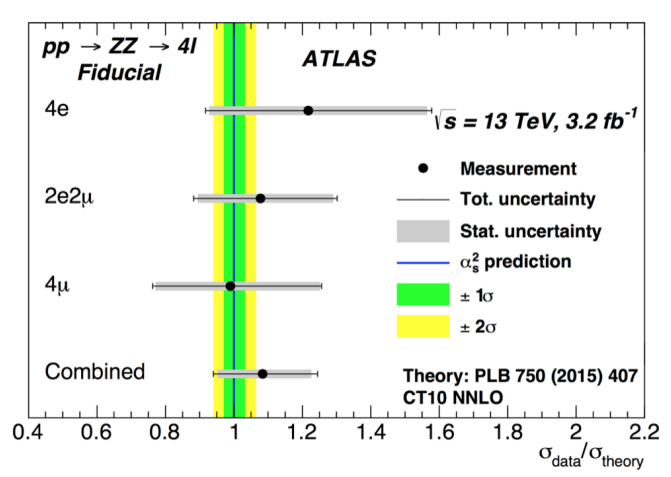

(a)

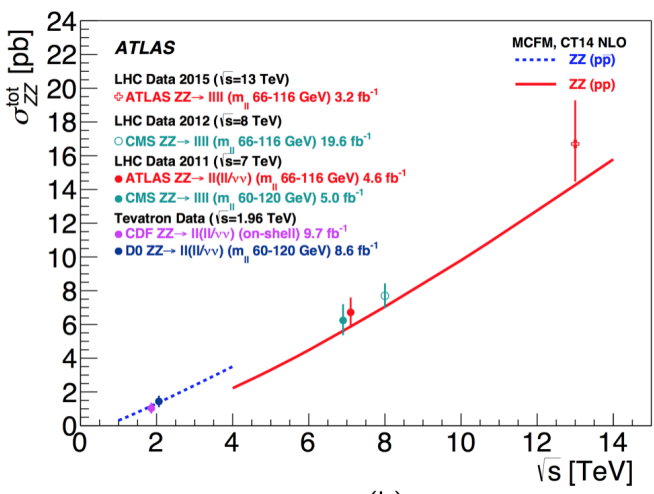

(b)

Figure 9: Fiducial cross-section measurements (a) for $Z Z$ production at $13 \mathrm{TeV}$ compared with the NNLO theoretical prediction indicated by the green and yellow band. The measurements are indicated by the black points, the statistical uncertainty is shown in grey and the total unceryainty is given by the error bar. Also shown is the $Z Z$ total cross-section measurement as a function of $\sqrt{s}$ (b). The $13 \mathrm{TeV}$ measurement discussed is shown by the right-most red point, the theoretical prediction is indicated by the red line. Other ATLAS and CMS measurements are indicated in red and turquoise respectively. The $p-\bar{p}$ prediction is given in blue with measurements from the Tevatron in purple and blue [11].

measurements agree well with the predictions. The measurements are currently statistically dominated, however the dominant systematic uncertainty is due to the determination of the non-prompt background.

Figure 11 shows the $W^{ \pm} Z$ total cross-section measurement as a function of $\sqrt{s}$. The measurements from the LHC are compared with both the NLO and NNLO predictions from Powheg. It is evident that the measurements all lie above the NLO prediction. The NNLO prediction, however, lies above the NLO prediction by around $10 \%$. This means that it can now be stated that the measurements documented in section 3 are in good agreement with the theoretical prediction.

Finally a first look at the differential distribution for the number of jets in the event $\left(N_{\text {jets }}\right)$ was 


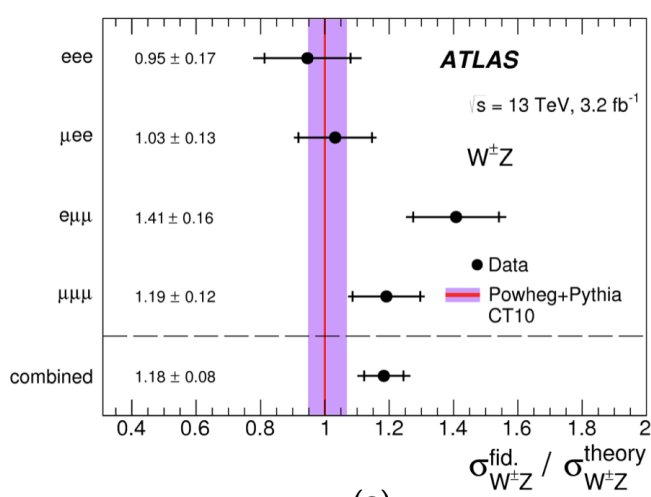

(a)

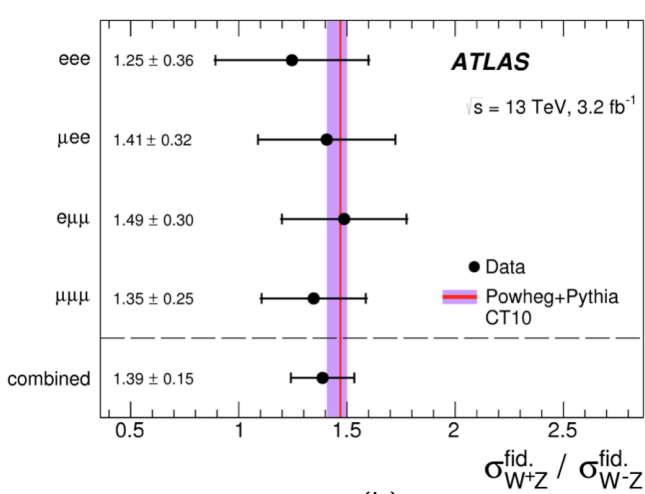

(b)

Figure 10: The $W^{ \pm} Z$ (a) and $W^{+} Z / W^{-} Z$ (b) fiducial cross-section measurements at $13 \mathrm{TeV}$ compared with the Powheg NLO theoretical predictions. The theoretical predictions are indicated by the purple band and the measurements are indicated by the black points and error bands [12].

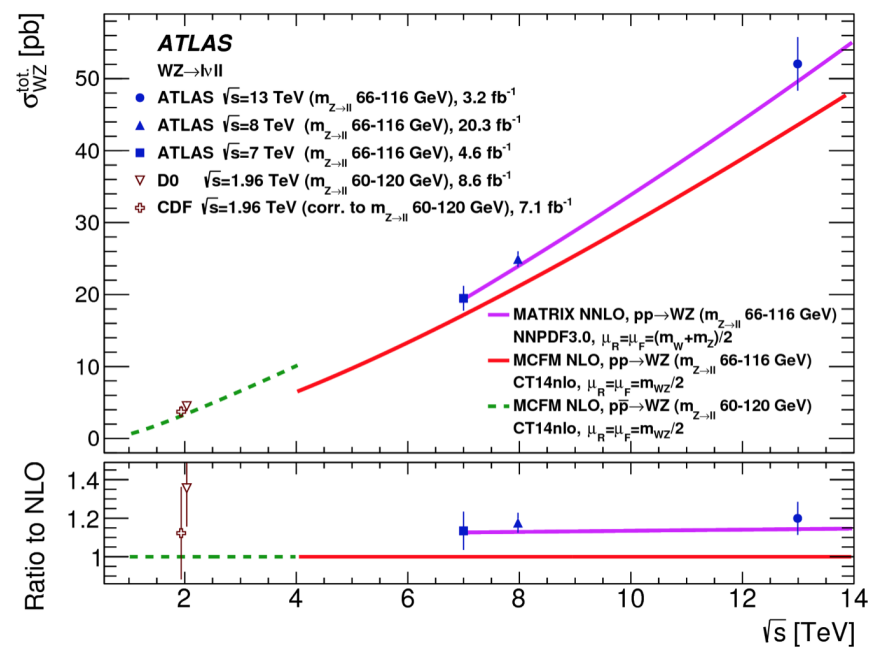

Figure 11: $W^{ \pm} Z$ total cross-section measurement as a function of $\sqrt{s}$. The $13 \mathrm{TeV}$ measurement discussed is shown by the right-most blue circular point, the theoretical prediction is indicated by the red line at NLO and in purple at NNLO. The other blue points indicate previous ATLAS measurements. The green dashed line indicates the $p-\bar{p}$ theoretical prediction and the hollow red points are measurements from the Tevatron experiments [12].

presented. This can be seen in Figure 12. For $N_{\text {jets }}<3$ good agreement is seen between the data and both MC predictions. Good agreement is also found between data and Sherpa at $N_{\text {jets }}>3$, as would be expected.

\section{Summary}

Measurements of di-boson and multi-boson production have been presented at both $8 \mathrm{TeV}$ and $13 \mathrm{TeV}$. Measurements of $W W, W Z, Z \gamma$ and $Z \gamma \gamma$ production at $\sqrt{s}=8 \mathrm{TeV}$ are presented along with measurements of $Z Z$ and $W Z$ production at $\sqrt{s}=13 \mathrm{TeV}$. All cross-section measurements are 


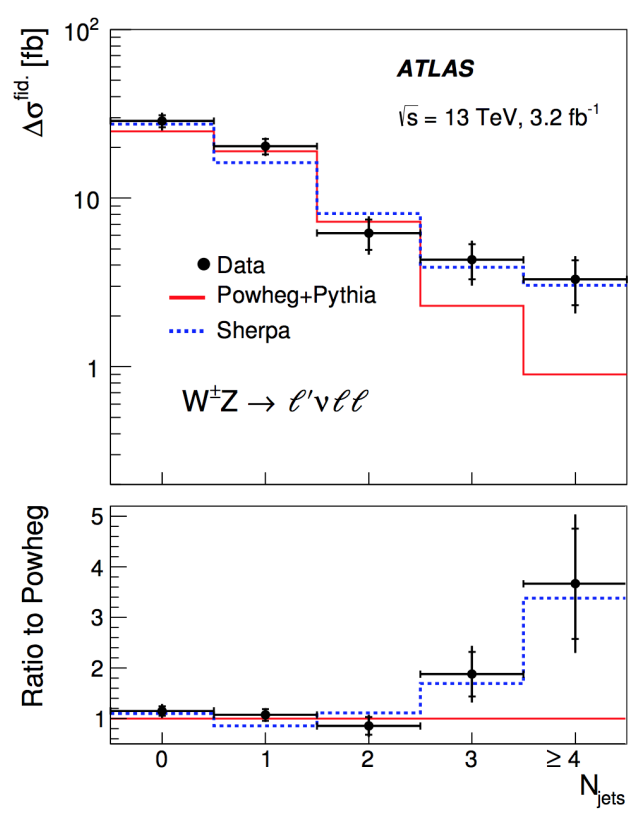

Figure 12: Differential distribution for the $N_{\text {jets }}$ distribution for $W^{ \pm} Z$ events at $13 \mathrm{TeV}$. The prediction from Powheg is shown in red and the prediction from Sherpa is shown in blue. The data is indicated by the black points and error bars. The lower panel shows the ratio of the data and Shepra to the Powheg prediction [12].

found to agree well with the corresponding theoretical predictions. Differential distributions have also been studied with a new theoretical precision. In general, good agreement is seen between the data and MC shapes. Finally, new $8 \mathrm{TeV}$ aTGC and aQGC measurements are found to be in good agreement with the SM. The limits presented are all improving upon or equaling the most stringent limits previously available. All analyses, however, stand to gain from the enhanced statistics of run-2.

\section{References}

[1] The ATLAS Collaboration, 2008 JINST 3, S08003.

[2] K. Hagiwara and R.D. Peccei and D. Zeppenfeld and K. Hikasa, Nucl. Phys. B, 282, (1987) 253.

[3] The ATLAS Collaboration, http://arxiv.org/abs/1603.01702

[4] The ATLAS Collaboration, http://arxiv.org/abs/1603.02151

[5] The ATLAS Collaboration, http://arxiv.org/abs/1208.1390

[6] The CMS Collaboration, http://arxiv.org/abs/1507.03268

[7] The D0 Collaboration, Phys. Rev. D 85, 112005, 2012

[8] The LEP Electroweak Working Group, http://arxiv.org/pdf/1302.3415v4.pdf

[9] M. Grazzini, S. Kallweit, D. Rathlev and M. Wiesemann, Phys. Lett. B, 761, (2016) 179-183.

[10] The ATLAS Collaboration, https://arxiv.org/abs/1604.05232v1

[11] The ATLAS Collaboration, Phys. Rev. Lett. 116, (2016) 101801

[12] The ATLAS Collaboration, https://arxiv.org/abs/1606.04017 